\title{
Immune response elicited by the oral administration of an intermediate strain of IBDV in chickens
}

\author{
Juan Manuel Carballeda ${ }^{1,2}$, Silvina Chimeno Zoth ${ }^{1,2}$, Evangelina Gómez ${ }^{1,2}$, \\ María Soledad Lucero $^{1,2}$, María José Gravisaco ${ }^{1}$ and Analía Berinstein ${ }^{1,2}$ \\ ${ }^{1}$ Instituto de Biotecnología, Instituto Nacional de Tecnología Agropecuaria, Buenos Aires, Argentina. \\ ${ }^{2}$ Consejo Nacional de Investigaciones Científicas y Técnicas, Buenos Aires, Argentina.
}

Submitted: November 1, 2013; Approved: March 14, 2014.

\begin{abstract}
The immune response elicited by the oral inoculation of an intermediate strain of infectious bursal disease virus was studied in chickens. A strong over expression of IL-6, IL-8, IFN $\alpha$ and IFN $\gamma$ was observed in bursa at 3 days post inoculation together with an increase in splenic $\mathrm{NO}_{2}$ release. An influx of T-lymphocytes was also detected.
\end{abstract}

Key words: Infectious Bursal Disease virus, innate immunity, avian cytokines, flow cytometry, RT-qPCR.

Infectious Bursal Disease Virus (IBDV), member of the Birnaviridae Family of the genus Avibirnavirus, is an endemic agent in most poultry producing areas worldwide. IBDV causes an acute, highly contagious, immunosuppressive disease in chickens (Eterradossi and Saif, 2008).

IBDV is a bisegmented double-stranded RNA virus whose genome is enclosed within a nonenveloped icosahedral capsid. The virus infects and destroys dividing IgM bearing B-lymphocytes. It has been demonstrated that both humoral and cellular immunosuppressions are observed in chickens infected with IBDV. Humoral immunosuppression is associated with the lysis of B-lymphocytes (Sharma et al., 1989). Cellular immunosuppression is evidenced by the ability of bursal $\mathrm{T}$ cells from IBDV infected chickens to inhibit concanavalin A (ConA)-mediated in vitro proliferation of normal splenocytes (Kim and Sharma, 2000). However, there is still need for discussion on the mechanism of this inhibition.

To date, two serotypes of the virus have been described. Serotype 1 IBDVs cause clinical signs and they are classified as mild, intermediate, intermediate plus, classical virulent and very virulent strains. Among them, mild, intermediate and intermediate plus viruses are used as live virus vaccines (Van den Berg, 2000). On the other hand, serotype 2 viruses may infect chickens and turkeys but they are non-pathogenic to both species (Jackwood et al., 1982; McFerran et al., 1980; McNulty and Saif, 1988).

IBDV infects chickens by the oral route and may initially replicate in cells of the gut-associated lymphoid tissues (Vervelde and Davison, 1997). Rautenschlein et al. (2003) compared the immunopathogenesis of different strains of IBDV. In their research, they included an intermediate strain (IBDV-B2), among other viruses studied, and they characterized the virus ability to replicate in bursal and extrabursal locations and to stimulate $\mathrm{B}$ and $\mathrm{T}$ cell immunity. Their study indicated that the ability of IBDV to induce $\mathrm{T}$ cell immunity may be important in protection and lasting immunologic memory and that extrabursal replication and persistence of the virus may determine the extent to which the cellular immune system gets stimulated (Rautenschlein et al., 2003). Eldaghayes et al. (2006) studied the bursal cytokine profile induced at early times by the intranasal inoculation of a classical virulent strain and a very virulent strain of IBDV and showed the induction of a pro-inflammatory response together with IFN $\gamma$ production. More recently, we developed a real time quantitative reverse transcription PCR assay to quantify the expression of different chicken cytokines and we characterized the cytokine profiles induced in bursa, spleen and duodenum of chickens intramuscularly (i.m.) inoculated with an intermediate strain of IBDV (Carballeda et al., 2011). In our previ- 
ous study, we found an inflammatory effect in different organs of chickens at short times after i.m. inoculation, showing similarity with previous results described for virulent IBDV strains (Carballeda et al., 2011). The aim of the present work was to study the immune response elicited by the oral inoculation of an intermediate strain of IBDV and to find out if the inoculation route influences the response observed.

Specific-pathogen-free White Leghorn chickens (eggs were purchased from Rosenbusch S.A., CABA, Argentina) were kept in individual cages with provision of food and water ad libitum. All procedures involving the use of animals were performed in agreement with institutional guidelines and approved by the Institutional Committee for the care and use of experimental animals (CICUAE CICVyA - INTA. Authorization reference number: 3/2011). Twenty four chickens of 3 weeks of age were randomly designated into two groups. The experimental group was orally inoculated with $200 \mu \mathrm{L}$ of an intermediate strain of IBDV [IBDV $10^{4}$ egg infectious dose ${ }_{50}\left(\mathrm{EID}_{50}\right)$, which is approximately 10 times greater than the vaccination dose; purchased from Laboratorios Inmuner, Entre Ríos, Argentina]. Chickens inoculated with an equal volume of sterile phosphate-buffered saline (PBS) were used as negative control (mock-inoculated group).

At 1, 3 and 5 days post inoculation (dpi), 3 chickens from each group were bled and euthanized. The 3 remaining birds of each group were bled weekly during a period of 28 days to measure specific antibodies against IBDV. At that time point, they also were sacrificed and their bursas processed. Thirty-milligram pieces of spleen, duodenum and bursa of Fabricius were excised from animals euthanized at 1, 3 and 5 dpi and kept immediately in RNAlater solution (QIAGEN, Valencia, CA) and RNA from each piece of tissue was obtained with the RNeasy kit (QIAGEN, Valencia, CA) according to the manufacturer's instructions. RNA was treated with DNAse I and reverse transcription was performed using SSIII Reverse transcription kit (Invitrogen, Carlsbad, CA) and random hexamers. Oligonucleotides used to amplify fragments of different chicken cytokines and glyceraldehyde 3-phosphate dehydrogenase (GAPDH) control genes were previously reported (Carballeda et al., 2011). For IL-1 $\beta$ amplification, primers IL-1 $\beta$ Fw: GGCTAACATTGCGCTGTAC and IL-1ßRv: CCCACTTAGCTTGTAGGTGGC were used.

Amplification and detection of mRNA levels were carried out using equivalent amounts of RNA from each tissue. Preparation of constructs and creation of standard curves for all cytokine genes used in this study, as well as for GAPDH gene, were performed as previously described (Carballeda et al., 2011).

The results obtained are shown in the Figure 1. One of the cytokines studied was IFN $\gamma$, which is a key Th1 cytokine that can activate macrophages to produce inflammatory factors such as IL-6 and iNOS, among other functions.
We found IFN $\gamma$ mRNA over-expressed (10 to 23 fold increase) mainly in spleen and bursa at $3 \mathrm{dpi}$. Also, plasmatic IFN $\gamma$ was measured by ELISA in plasma samples of treated chickens (CytoSet Kit, Biosource, CA, USA) and it could be detected at 1 and 3 dpi in IBDV-inoculated animals (data not shown); however, the highest levels were observed at 3 dpi. Concomitantly, oral administration of IBDV induced a mild upregulation of the proinflammatory cytokine IL-6 in spleen at 1, 3 and 5 dpi and in duodenum at 3 dpi. By contrast, a very strong upregulation (200-fold increase) of this cytokine was observed in bursa at 3 dpi.

The chemokine IL-8, which serves as a chemical signal that attracts heterophils to the site of inflammation, accompanied the strong IL- 6 and IFN $\gamma$ mRNA upregulation found in bursa of IBDV-treated chickens at 3 dpi. Unexpectedly, IL-1 $\beta$, another pro-inflammatory cytokine, was not strongly upregulated (only a slight upregulation was observed in the spleen of IBDV-inoculated chickens at 1 and $3 \mathrm{dpi}$ ). This result is opposite to the findings of Khatri and coworkers (2005) who showed that virulent IBDV (IM strain) infected chickens suffered a strong IL-1 $\beta$ upregulation (65-fold) at $3 \mathrm{dpi}$ in bursal macrophages. LITAF, a transcription factor associated with TNF $\alpha$, was 6 fold upregulated only in the bursa of IBDV-treated animals at $5 \mathrm{dpi}$. Overall, we observed that the cytokine production modifications when using the oral route were detected at later times than when animals were inoculated by the i.m. route (Carballeda et al., 2011). This fact could be due to an earlier availability of the virus and the concomitant induction of the immune response when using the i.m. route.

Previous reports demonstrated an absence of upregulation of IFN $\alpha$ when chickens were infected with classical virulent and very virulent IBDV strains (Eldaghayes et al., 2006). In another study, Rauf et al. (2011) showed that the oral inoculation of IBDV classical strains promotes a downregulation of IFN $\alpha$ mRNA at 3 dpi in the bursa, but variant strains (that produce less pronounced bursal damage, inflammatory response and infiltration of $\mathrm{T}$ cells than classical strains) promote an upregulation of this gene at the same time point. In accordance with Rauf et al. (2011), we found an upregulation of IFN $\alpha$ in duodenum and bursa at 3 dpi, which, in our work, was also detected at 1 dpi. This result could partially explain the difference in the virulence degree between strains as animals infected with high virulent strains seem to fail in the production of IFN $\alpha$, a very important antiviral cytokine. In a recent work, $\mathrm{Li}$ et al. (2013) demonstrated that VP4 viral protein from a virulent strain of IBDV caused suppression of type 1 IFN expression by interacting with GILZ protein of host cells. In the present study, the animals treated with an intermediate strain of IBDV were able to produce IFN $\alpha$, probably involved in the interruption of viral dissemination.

Chicken IL-15 was shown to act as a T cell growth factor (Lillehoj et al., 2001). We observed an increment in 
IL-15 levels in spleen, bursa and duodenum, mainly at 5 dpi. In general, IL-15 mRNA expression is increased when microbial activators of macrophages are present (Doherty et al., 1996). As IBDV infects and replicates in macrophages leading to the production of pro-inflammatory and Th1 cytokines (Khatri and Sharma, 2006) selective up regulation of IL-15 could have been enhanced by the presence of the virus.

Another piece of the same spleens harvested aseptically were used for $\mathrm{NO}_{2}$ assay, since splenic nitrite production is considered a macrophage activation marker (Jeurissen et al., 2000). Briefly, splenocytes were resuspended in RPMI medium supplemented with $2 \mathrm{mM}$ L-glutamine, $100 \mathrm{U} / \mathrm{mL}$ penicillin, $100 \mu \mathrm{g} / \mathrm{mL}$ streptomycin, $10 \mathrm{mM}$ HEPES, $50 \mu \mathrm{M}$ 2-mercaptoethanol and 10\% fetal bovine serum (FBS). One million cells per well were seeded on 96 well-culture plates in the presence or absence of ConA $(5 \mathrm{mg} / \mathrm{mL})$ and incubated for $24 \mathrm{~h}$ at $41{ }^{\circ} \mathrm{C}$ in a $5 \% \mathrm{CO}_{2}$ atmosphere. Culture supernatants were collected and nitrite concentration was measured by the Griess reaction (Tsikas, 2007). When compared to PBS-inoculated chickens, IBDV-treated birds showed a significant increase $(\mathrm{p}<0.05)$ in $\mathrm{NO}_{2}$ production in both ConA- $(26.03 \pm 1.21$ against 9.20 $\left.\pm 0 \mu \mathrm{M} \mathrm{NO}_{2}\right)$ and mock-stimulated (14.48 \pm 0.20 against $4.21 \pm 0.20 \mu \mathrm{M} \mathrm{NO}_{2}$ ) splenocytes at $1 \mathrm{dpi}$ and only in ConA-stimulated $\left(33.3 \pm 2.42\right.$ against $\left.6.99 \pm 0.30 \mu \mathrm{M} \mathrm{NO}_{2}\right)$ splenocytes at $3 \mathrm{dpi}$. We have previously demonstrated that ConA-stimulated splenocytes obtained from chickens inoculated i.m. with the same strain of IBDV, at 3 and $5 \mathrm{dpi}$, ex-
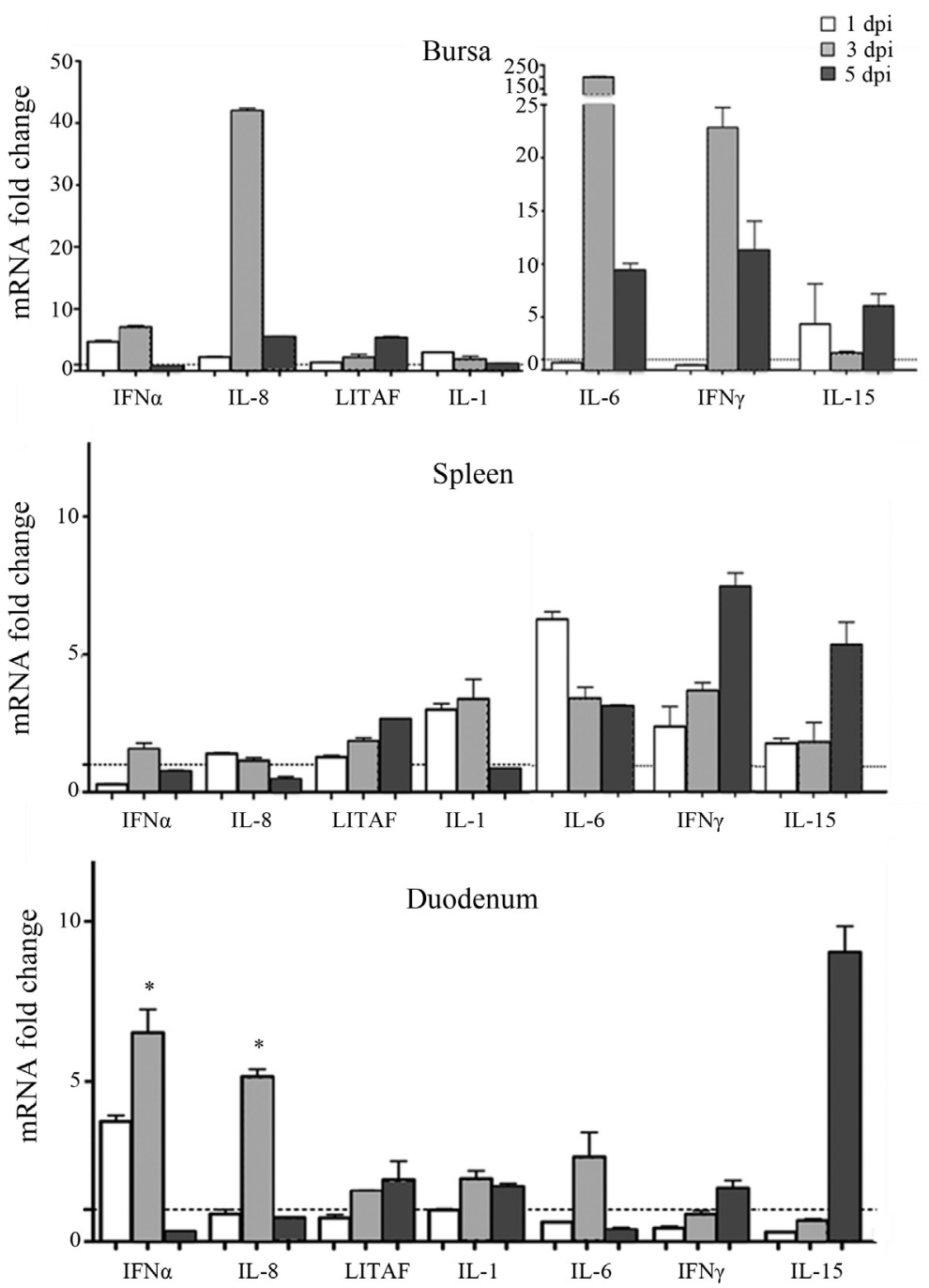

Figure 1 - Transcriptional pattern of cytokine genes. Total RNA was extracted from bursa, spleen and duodenum of IBDV-or mock-inoculated chickens at 1, 3 and 5 dpi and cDNA was synthesized. mRNA levels were determined by Quantitative Real Time PCR using specific primers and SYBR ${ }^{\circledR}$ Green method. The expression level of each mRNA was calculated in relation to the expression level of GAPDH gene. Each bar represents the ratio of the mean \pm SEM of 2 replicates of each sample obtained from pools of 3 IBDV-inoculated chickens and the mean \pm SEM of 2 replicates of each sample obtained from pools of 3 mock-inoculated chickens. The Students $t$ test was used to determine significant differences between mock-infected and IBDV-infected chickens. A value of $\mathrm{p}<0.05$ was considered to be statistically significant. 
Table 1 - Evaluation of cell populations by flow cytometry.

\begin{tabular}{|c|c|c|c|c|c|}
\hline \multirow[t]{2}{*}{ Days post infection } & \multicolumn{5}{|c|}{$\%$ Positive cells in the gate } \\
\hline & Source of cells & CD3+ & $\mathrm{CD}^{+} \mathrm{CD}^{+}$ & $\mathrm{CD}^{+} \mathrm{CD} 8 \alpha^{+} \beta^{+}$ & $\mathrm{CD}^{-} \mathrm{CD} 8 \alpha^{+}$ \\
\hline \multirow[t]{2}{*}{ 1dpi } & Mock & 2.71 & 0.94 & 0.23 & 0.22 \\
\hline & IBDV & 3.69 & 0.85 & 0.32 & 0.22 \\
\hline \multirow[t]{2}{*}{$5 \mathrm{dpi}$} & Mock & 1.43 & 0.50 & 0.19 & 0.60 \\
\hline & IBDV & 10.83 & 4.80 & 5.10 & 2.46 \\
\hline \multirow[t]{2}{*}{ 28dpi } & Mock & 1.56 & 0.67 & 0.33 & 0.44 \\
\hline & IBDV & 1.59 & 0.66 & 0.60 & 0.42 \\
\hline
\end{tabular}

Chicken leukocytes from pools of bursas of IBDV- or Mock-inoculated animals (1, 5 and 28 dpi) were stained with different combinations of antibodies and analyzed by flow cytometry. Lymphocyte population was gated according to their size and granularity. Results were expressed as frequency of positive cells in the gate.

hibited less $\mathrm{NO}_{2}$ production than stimulated splenocytes from control animals. In the same way, we observed macrophage population decay in spleen (Carballeda et al., 2011). This difference could be due to the way each immunization route acts over the immune system.

Remaining bursas were used to study mononuclear cell populations by flow cytometry as described by Carballeda et al. (2011). Briefly, bursas were cut and mechanically disrupted in RPMI 1640. Then, cellular suspensions were passed through a $40 \mu \mathrm{m}$ mesh (Cell Strainer, BD) and mononuclear cells were isolated by centrifugation over Histopaque density gradient. Cells were recovered from the interface, washed, and live cells were counted using trypan blue exclusion. Subsequently, cells were diluted in staining buffer (PBS 1x, 10\% FBS, 0.1\% Sodium Azide) and 1x10 cells per well were seeded on 96 well- plates (V-shape) and washed twice with the same buffer. Staining was performed by resuspending cells of each well with $100 \mu \mathrm{L}$ of staining buffer containing different combinations of antibodies or individual ones as single-color staining for compensation. Monoclonal antibodies (mAbs) (CD3-SPRD, CD4-PE, CD8 $\alpha$-FITC, CD8 $\beta$-PE) were purchased from Southern Biotech. (Birmingham, AL). Cell suspensions were analyzed with a FACS Calibur flow cytometer (BD Biosciences, San Jose, CA) and CellQuest software. The lymphocyte gate was defined by the forward/side scatter characteristics of the cells and 30,000 events were analyzed for each sample. Results are presented in the Table 1. At 1 and 3 dpi, no changes were observed in bursal T-lymphocytes population. However, at 5 dpi a notable infiltration of T lymphocytes was observed in the bursa of animals inoculated with IBDV. The table shows the frequency of total $\mathrm{CD}^{+}, \mathrm{CD}^{+}$and $\mathrm{CD} 8 \alpha^{+} \beta^{+}$lymphocytes revealing an important increase in the bursa of IBDV treated animals. Similar results were obtained when the virus was inoculated by i.m. route (Carballeda et al., 2011), suggesting that the virus causes the same effect when arrives at bursa independently of the inoculation route. In ad- dition, $\mathrm{T}$ lymphocytes values returned to the basal level at 28 dpi.

Animals sacrificed at 28 dpi were bled weekly in order to measure specific antibodies against IBDV. Samples were analyzed using FlowChek IBDV Kit (IDEXX, Maine, USA) following the manufacturer's instructions. IBDV treatment promoted an adequate adaptive immune response evidenced by a gradual increase in the anti-IBDV antibodies titers, reaching the highest value at $28 \mathrm{dpi}(2384 \pm 888$ compared to $110 \pm 154$ in the mock-infected group, being this last value negative for the used assay).

The comparison of our data with the results reported by others demonstrates that different strains of IBDV have, overall, the same effect on the response parameters studied (i.e., an inflammatory effect in different organs of chickens at short times after inoculation). Nevertheless, the degree of virulence and harm produced by the different pathotypes is noticeable. Since IFN $\alpha$ is a strong antiviral agent, the ability of the intermediate strain of IBDV to induce the production of IFNa could contribute to the dissemination restriction of this virus. However, we cannot rule out other factors contributing to its reduced virulence compared to classical virulent and very virulent IBDV strains. The present work contributes to augment the knowledge about the immune parameters involved in the response of chickens to infection with an intermediate strain of IBDV.

\section{Acknowledgments}

Authors want to acknowledge Julia Sabio y García for excellent English language editing, and Silvio Díaz for his animal husbandry work. This work was supported by grant PE 232152 from INTA.

\section{References}

Carballeda JM, Chimeno Zoth S, Gomez E, Gravisaco MJ, Berinstein A (2011) Activation of the immune response against Infectious Bursal Disease Virus after intramuscular 
inoculation of an intermediate strain. Immunobiol 216:1028-1033.

Doherty TM, Seder RA, Sher A (1996) Induction and regulation of IL-15 expression in murine macrophages. J Immunol 156:7 35-741.

Eldaghayes I, Rothwell L, Williams A, Withers D, Balu S, Davison F, Kaiser P (2006) Infectious bursal disease virus: strains that differ in virulence differentially modulate the innate immune response to infection in the chicken bursa. Viral Immunol 19:83-91.

Eterradossi N and Saif YM (2008) Infectious Bursal Disease. In: Saif, Y.M., Fadly, A.M., Glisson, J.R., McDougald, L.R., Nolan, L.K., Swayne, D.E. (eds) 12th edition. Diseases of Poultry. Blackwell Publlishing, Ames, pp 185-208.

Jackwood DJ, Saif YM, Hughes JH (1982) Characteristics and serologic studies of two serotypes of infectious bursal disease virus in turkeys. Avian Dis 26:871-882.

Jeurissen SH, Boonstra-Blom AG, Al-Garib SO, Hartog L, Koch G (2000) Defense mechanisms against viral infection in poultry: a review. Vet Q 22:204-208.

Khatri M, Palmquist JM, Cha RM, Sharma JM (2005) Infection and activation of bursal macrophages by virulent infectious bursal disease virus. Virus Res 113:44-50.

Khatri M and Sharma JM (2006) Modulation of macrophages by infectiousbursal disease virus. Cytogenet. Genome Res 117:388-393.

KimIJ,SharmaJM (2000) IBDV-induced bursal T lymphocytes inhibit mitogenic response of normal splenocytes. Vet Immunol Immunopathol 754:47-57.

Li Z, Wang Y, Li X, Li X, Cao H, Zheng SJ (2013) Critical roles of glucocorticoid-induced leucine zipper in infectious bursal disease virus (IBDV)-induced suppression of type I Interferon expression and enhancement of IBDV growth in host cells via interaction with VP4. J Virol 87:1221-1231.

Lillehoj HS, Min W, Choi KD, Babu US, Burnside J, Miyamoto T, Rosenthal BM, Lillehoj EP (2001) Molecular, cellular, and functional characterization of chicken cytokines homol- ogous to mammalian IL-15 and IL-2.Vet. Immunol Immunopathol 82:229-244.

McFerran JB, McNulty MS, McKillop ER, Connor TJ, McCracken NRM, Collings DS, Allan GM (1980) Isolation and serological studies with infectious bursal disease viruses from fowl, turkeys and ducks: demonstration of a second serotype. Avian Pathol 9:395-404.

McNulty MS, Saif YM (1988) Antigenic relationship of nonserotype 1 turkey infectious bursal disease viruses from the United States and United Kingdom. Avian Dis 32:374-375.

Rauf A, Khatri M, Murgia MV, Jung K, Saif YM (2011) Differential modulation of cytokine, chemokine and Toll like receptor expression in chickens infected with classical and variant infectious bursal disease virus. Vet Res 42:85.

Rautenschlein S, Yeh HY, Sharma JM (2003) Comparative immunopathogenesis of mild, intermediate and virulent strains of Classic Infectious Bursal Disease Virus. Avian Dis 47:66-78.

Sharma JM, Dohms JE, Metz AL (1989) Comparative pathogenesis of serotype 1 and variant serotype 1 isolates of infectious bursal disease virus and their effect on humoral and cellular immune competence of specific-pathogen-free chickens. Avian Dis 33:112-124.

Tsikas D (2007) Analysis of nitrite and nitrate in biological fluids by assays based on the Griess reaction: appraisal of the Griess reaction in the L-arginine/nitric oxide area of research. J. Chromatogr. B Analyt.Technol.Biomed. Life Sci 851:51-70.

Van den Berg TP (2000) Acute infectious bursal disease in poultry: a review. Avian Pathol 29:17.

Vervelde L, Davison TF (1997) Comparison of the in situ changes in lymphoidcells during infection with infectious bursal disease virus in chickens of different ages. Avian Pathol 26:803-821.

All the content of the journal, except where otherwise noted, is licensed under a Creative Commons License CC BY-NC. 